\title{
Multi-physics Properties in Ferroelectric Nanowires and Related Structures from First-principles
}

\author{
Takahiro Shimada and Takayuki Kitamura \\ Department of Mechanical Engineering and Science, Kyoto University
}

Japan

\section{Introduction}

Ferroelectric (FE) nanowires (Spanier et al., 2006) have drawn much attention as onedimensional (1D) multifunctional materials for their technological applications, e.g., ferroelectric random access memories (FeRAM) (Scott, 2000). In recent years, singlecrystalline $\mathrm{PbTiO}_{3}$ nanowires with a diameter of 5-12 nm (Gu et al., 2007) have been manufactured, and smaller nanowires with several lattice spacings are anticipated in the near future. The ferroelectric nanowires are surrounded by both surfaces and atomically sharp edges consisting of (100) and (010) surfaces (Yamashita et al., 2002). The edge structure is commonly observed in nanostructured perovskite-type oxides, e.g., the step on the $\mathrm{SrTiO}_{3}$ (001) surface (Cho et al., 2004), the zigzag (110) surface structure (Kotomin et al., 2004), and the edge of $\mathrm{PbTiO}_{3}$ nano-island (Chu et al., 2004). Thus, the edges are among the characteristic nanostructures in perovskite oxides.

Ferroelectricity is defined as a property that materials exhibit non-vanishing spontaneous polarization, which is a dipole moment per unit volume, even in the absence of external electric fields. Figure 1 shows the crystal structures of perovskite oxides $\mathrm{ABO}_{3}$ in the paraelectric (PE) and ferroelectric (FE) phases. In the FE phase where atoms are displaced in a specific direction from their ideal lattice sites, spontaneous polarization emerges due to its non-symmetrical atomic structure, while there is no polarization in the PE phase where the structure is centro-symmetric. The FE-phase structure is energetically favorable, and it has, in general, two equivalent states with upward and downward spontaneous polarization, $\boldsymbol{P}_{\mathrm{UP}}$ and $\boldsymbol{P}_{\mathrm{DOWN}}$, respectively. They are switchable each other by applied external electric fields.

The rapid change in coordination number at the surface and edge can significantly affect the ferroelectric distortions in the nanowires because ferroelectricity originates from the delicate balance between the short-range covalent and long-range Coulomb interactions. In addition, nanowires are normally subjected to axial tension or compression, which sometimes enhances or destabilizes the ferroelectric distortions. Such a coupling effect between mechanical deformation and electric properties is known as "multi-physics properties" and it is of importance for the design of nanoscale materials. For example, strained silicon (Chakrabarti et al., 2001) is one of the well-known representatives leveraging the multiphysics property: Applied strain changes the band structure of silicon, and at the critical 
strain silicon undergoes the semiconducting - metallic transition (Umeno et al., 2002). Such multi-physics properties have been also intensively studied in the ferroelectric or ferromagnetic materials. The mechanical deformation not only changes the electronic polarization but also induces the ferroelectric-paraelectric phase transition.

First-principles (ab initio) density functional theory calculations (Hohenberg \& Kohn, 1964) (Kohn \& Shan, 1965), which can analyze the electronic ground-state as well as atomic structure by solving the Schrödinger equation within the one-electron approximation, are the powerful tool for the study of multi-physics properties. The method has been applied to various kinds of materials, for example, the calculations have revealed the characteristic change in ferroelectric properties (including its disappearance) of nano-structured ferroelectrics, such as nanofilms induced by applied strain.

In this chapter, we demonstrate the characteristic ferroelectricity at the surfaces and edges in $\mathrm{PbTiO}_{3}$ nanowires, and the crucial role of mechanical strain, namely, multi-physics properties from the atomistic and electronic points of view using first-principles (ab initio) density-functional theory calculations.

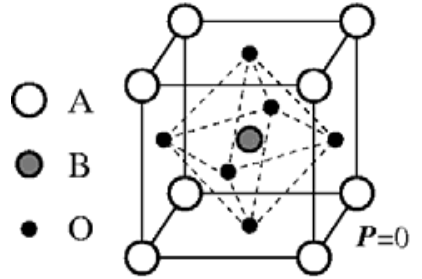

Paraelectric (PE)
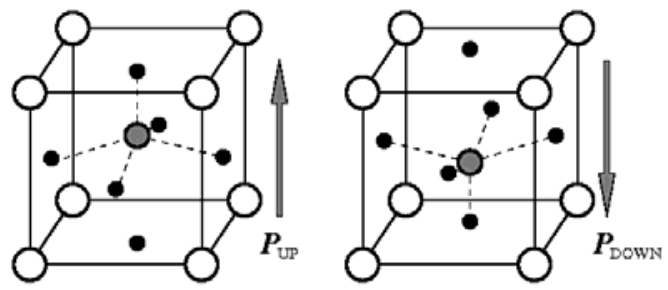

Ferroelectric (FE)

Fig. 1. Atomic structures of perovskite oxides $\mathrm{ABO}_{3}$ in the paraelectric (PE) phase and ferroelectric (FE) phase with upward and downward polarization.

\section{First-principles ( $a b$ initio) density functional theory calculations}

The description of macroscopic solids from first-principles is based on the determination of the quantum mechanical ground-state associated to their constituting electrons and nuclei. It consists in calculating the quantum mechanical total energy of the system and in the subsequent minimization of that energy with respect to the electronic and nuclear coordinates (variational principle). This defines a complex many-body problem of interacting particles. Density Functional Theory (DFT), proposed in the 1960's by Hohenberg and Kohn (Hohenberg \& Kohn, 1964) and Kohn and Sham (Kohn \& Shan, 1965), provided a simple method for describing the effect of electron-electron interactions. Hohenberg and Kohn first proved that the total energy of an electron gas is a unique functional of the electron density. This means that instead of seeking directly for the complex many-body wave function of the system, we can adopt an intrinsically different point of view and consider the electronic density as the fundamental quantity of the problem. The minimum value of the total energy density functional is the ground-state energy of the system and the density yielding this minimum value is the exact ground-state density.

According to the density functional theory (DFT) (Hohenberg \& Kohn, 1964) (Kohn \& Shan, 1965), the Kohn-Sham total-energy functional in the presence of a static external potential, $v(\boldsymbol{r})$, can be written as a functional of the electronic density, $\rho(\boldsymbol{r})$, 


$$
E_{t o t}=\int v(\mathbf{r}) \rho(\mathbf{r}) d \mathbf{r}+T[\rho(\mathbf{r})]+\frac{1}{2} \iint \frac{\rho(\mathbf{r}) \rho\left(\mathbf{r}^{\prime}\right)}{\left|\mathbf{r}-\mathbf{r}^{\prime}\right|} d \mathbf{r}^{\prime} d \mathbf{r}+E_{x c}[\rho(\mathbf{r})]
$$

where the first, second, third and fourth terms denote the static total external (or electronion) potential energy, the kinetic energy of the electrons, the Coulomb energy associated with interactions among the electrons and the exchange-correlation energy, respectively. It is necessary to determine the set of wave functions, $\psi_{i}(\boldsymbol{r})$, that minimize the Kohn-Sham energy functional. These are given by the self-consistent solutions to the Kohn-Sham equations,

$$
\left[-\frac{1}{2} \nabla^{2}+v_{e f f}(\mathbf{r})\right] \psi_{i}(\mathbf{r})=\varepsilon_{i} \psi_{i}(\mathbf{r}),
$$

where $\psi_{i}(\boldsymbol{r})$ is the wave function of electronic state $i, \varepsilon_{i}$ is the Kohn-Sham eigenvalue, and $v_{\text {eff }}(\boldsymbol{r})$ is the effective one-electron potential which is given by

$$
v_{e f f}(\mathbf{r})=v(\mathbf{r})+V_{\text {coul }}(\mathbf{r})+\varepsilon_{x c}(\mathbf{r})
$$

where $V_{\text {coul }}(\boldsymbol{r})$ and $\varepsilon_{x c}(\boldsymbol{r})$ denote the Coulomb potential of electrons and the exchangecorrelation potential, respectively. They can be written

$$
V_{\text {coul }}(\mathbf{r})=\int \frac{\rho(\mathbf{r})}{\left|\mathbf{r}-\mathbf{r}^{\prime}\right|} d \mathbf{r}^{\prime}
$$

and

$$
\varepsilon_{x c}(\mathbf{r})=\frac{\partial E_{x c}[\rho(\mathbf{r})]}{\partial \rho(\mathbf{r})}
$$

The electronic density, $\rho(\boldsymbol{r})$, is given by

$$
\rho(\mathbf{r})=2 \sum_{i}^{o c c}\left|\psi_{i}(\mathbf{r})\right|^{2}
$$

The Kohn-Sham equations must be solved self-consistently so that the occupied electronic states generate a charge density that produces the electronic potential that was used to construct the equations.

It has been validated that first-principles calculations can accurately predict not only atomic structures in solids, at surfaces, domain walls and grain boundaries but also the electronic properties (band structure, magnetic moment, spontaneous polarization and conductivity). For ferroelectrics, atomic structures, ferroelectric characters, mechanical and vibrational properties are also well reproduced by the first-principles calculations.

\section{Ferroelectricity and multi-physics properties at surfaces}

\subsection{Simulation procedure and models}

Figure 2 shows simulation models for the study of $\mathrm{PbTiO}_{3}(001)$ surface with both $\mathrm{TiO}_{2}$ - and PbO-terminations with a $(1 \times 1)$ periodicity. Each slab model has 9 atomic layers. The three dimensional periodic boundary conditions are applied for all the models. The thickness of 
the vacuum regions are set to $12 \AA$ so that undesirable interaction from imaginary films does not appear.

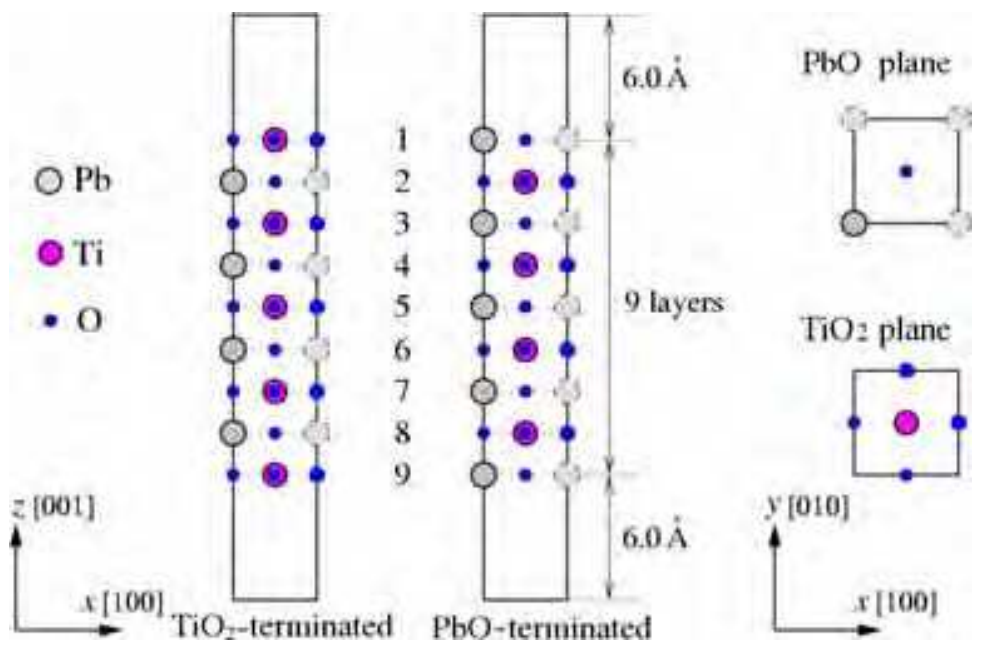

Fig. 2. Simulation models of $\mathrm{PbTiO}_{3}(001)$ surface of $\mathrm{TiO}_{2}$ - and $\mathrm{PbO}$-termination with a $(1 \times 1)$ periodicity. Solid boxes represent simulation cells.

$A b$ initio calculations based on the projector augmented wave (PAW) method (Blochl, 1994) are conducted using the VASP (Vienna Ab-initio Simulation Package) code (Kresse \& Hafner, 1993) (Kresse \& Furthmuller, 1996). The plane-wave cutoff energy is set to $500 \mathrm{eV}$. The local density approximation (LDA) of the Ceperley-Alder form (Ceperley \& Alder, 1980 ) is used for the evaluation of the exchange correlation energy. The pseudopotentials include the $\mathrm{O} 2 \mathrm{~s}$ and $2 \mathrm{p}$, the Ti $3 \mathrm{~s}, 3 \mathrm{p}, 3 \mathrm{~d}$ and $4 \mathrm{~s}$, the $\mathrm{Pb} 5 \mathrm{~d}, 6 \mathrm{~s}$ and $6 \mathrm{p}$ electrons in the valence states. The Brillouin-zone integrations are carried out with a $4 \times 4 \times 2$ Monkhorst-Pack (Monkhorst \& Pack, 1976) $k$-point mesh. Structural relaxation is performed by means of the conjugate gradient method until all the forces acting on atoms are less than $0.005 \mathrm{eV} / \AA$.

Initially the atoms are arranged on the lattice points of the cubic perovskite structure with its theoretical lattice constant of $a_{0}=3.891 \AA$. Then, the atomic coordinates are fully relaxed with a centrosymmetry in the z-direction to confine the ferroelectricity only in the lateral directions. In this study, (a) in-plane isotropic $\left(\varepsilon_{x x}=\varepsilon_{y y} \neq 0\right)$ and (b) [100]-uniaxial $\left(\varepsilon_{x x} \neq 0, \varepsilon_{y y}\right.$ $=0$ ) strain conditions are simulated (see also Fig. 3). On both cases, a small strain is applied stepwise and atomic coordinates are fully relaxed at each straining step.

\section{In-plane isotropic strain Uniaxial strain along [100]}
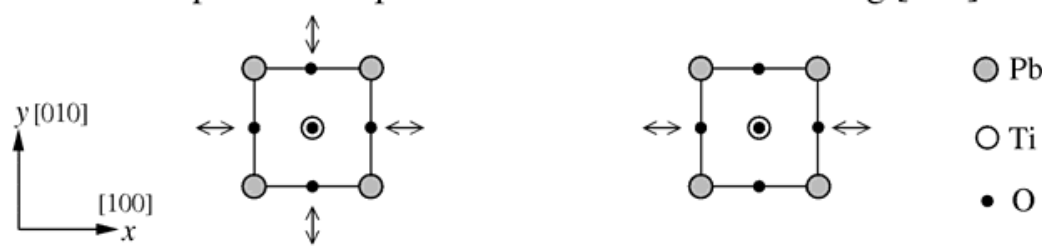

Fig. 3. Schematic illustration explaining the two types of deformation of the in-plane isotropic strain (left side) and uniaxial strain along the [100] (right side). 


\subsection{Ferroelectricity of unstrained surface structures}

We introduce $\delta$ defined as follows to represent strength of polarization distortion of each layer,

$$
\begin{gathered}
\delta_{i}=\left\{\begin{array}{lll}
{\left[\bar{\delta}_{i}(\mathrm{~Pb})-\overline{\delta_{i}}(\mathrm{O})\right]} & (\mathrm{PbO} & \text { layer }) \\
{\left[\bar{\delta}_{i}(\mathrm{Ti})-\bar{\delta}_{i}(\mathrm{O})\right]} & \left(\mathrm{TiO}_{2}\right. & \text { layer })
\end{array} \quad(i=x, y)\right. \\
\delta=\sqrt{\delta_{x}^{2}+\delta_{y}^{2}}
\end{gathered}
$$

where $\delta_{i}$ is the layer-averaged atomic displacement relative to ideal lattice sites (see also Fig. 4). We simulated the [100]- and [110]-polarized films and found that the polarization in [110] direction is preferred with a small energy advantage of $2.96 \mathrm{~mJ} / \mathrm{m}^{2}$ over [100] polarization in both $\mathrm{TiO}_{2-}$ and PbO-terminations. The layer-by-layer $\delta$ of both the [100]- and [110]polarized films is shown in Table 1 although the [110]-polarized case is more stable. The $\delta$ gradually decreases toward the surface layer for the $\mathrm{TiO}_{2}$-termination, while the opposite trend is found in the PbO-termination. The magnitude of $\delta$ at Layer 5 is close to that of the bulk value for both terminations indicating that our models have sufficient thickness to avoid the effect from another surface.

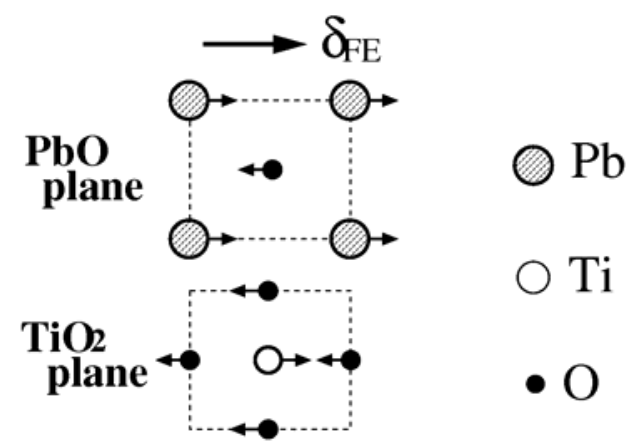

Fig. 4. Schematic illustration explaining the structural parameter of polarization distortion, $\delta$.

\subsection{Influence of in-plane isotropic tension/compression}

The polarization distortion per area must be the relevant value to represent the response of polarization to strain because polarization of bulk material is defined as dipole moment per volume. Change in the polarization distortion per area, $\delta^{\prime}=\delta / A(A$ is the cross-sectional area of simulation cell in the $x y$ direction), with respect to in-plane isotropic strain is shown in Fig. 5. Here, the polarization direction is always in [110] direction because we confirmed that [100]-polarized structure has larger energy under nonzero isotropic strain as well. In the case of $\mathrm{TiO}_{2}$-termination, $\delta$ increases smoothly with increasing tensile strain, showing the enhancement of ferroelectricity by tangential stretch. On the other hand, ferroelectricity is suppressed under compression and vanishes at $\varepsilon=-0.06$, indicating the existence of the critical compressive strain for ferroelectricity. When the surface is further compressed the ferroelectricity emerges again. The response of $\delta$ to the tangential strain in the $\mathrm{PbO}-$ terminated surface is similar. However, under compression, $\delta$ does not vanish because of the enhancement of FE distortion by PbO-termination. 


\begin{tabular}{|c|c|c|c|c|c|c|c|c|}
\hline \multirow{2}{*}{} & \multicolumn{3}{|c|}{$\mathrm{TiO}_{2}$ termination } & \multicolumn{3}{c|}{ PbO termination } \\
\cline { 2 - 9 } & \multicolumn{2}{|c|}{$\mathrm{P}[100]$} & \multicolumn{2}{c|}{$\mathrm{P}[110]$} & \multicolumn{2}{c|}{$\mathrm{P}[100]$} & \multicolumn{2}{c|}{$\mathrm{P}[110]$} \\
\hline Layer & $\mathrm{TiO}_{2}$ & $\mathrm{PbO}$ & $\mathrm{TiO}_{2}$ & $\mathrm{PbO}$ & $\mathrm{TiO}_{2}$ & $\mathrm{PbO}$ & $\mathrm{TiO}_{2}$ & $\mathrm{PbO}$ \\
\hline 1 & 2.29 & & 2.58 & & & 13.48 & & 12.10 \\
\hline 2 & & 3.40 & & 3.64 & 4.75 & & 5.26 & \\
\hline 3 & 3.48 & & 3.82 & & & 8.77 & & 8.70 \\
\hline 4 & & 6.77 & & 6.62 & 4.09 & & 4.34 & \\
\hline 5 & 3.81 & & 4.04 & & & 8.05 & & 7.93 \\
\hline Bulk & 3.95 & 7.67 & 4.14 & 7.47 & 3.95 & 7.67 & 4.14 & 7.47 \\
\hline
\end{tabular}

Table 1. Polarization distortion, $\delta$, in percentage of the lattice parameter $\left(a_{0}=3.891 \AA\right)$ at the $\mathrm{TiO}_{2}$ - and $\mathrm{PbO}$-terminated surfaces with a $(1 \times 1)$ periodicity.

(a) $\mathrm{TiO}_{2}$ termination

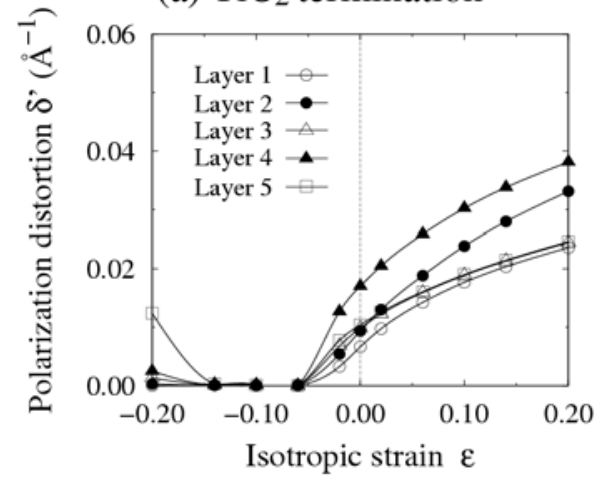

(b) $\mathrm{PbO}$ termination

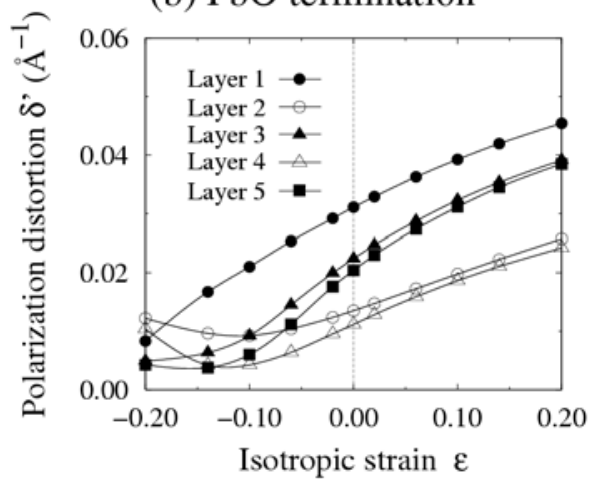

Fig. 5. Polarization distortion per area, $\delta^{\prime}$, as a function of in-plane isotropic strain, $\varepsilon$, in the $(1 \times 1)$ model with (a) $\mathrm{TiO}_{2}$ and (b) $\mathrm{PbO}$ termination.

\subsection{Effect of uniaxial tension/compression}

Tensile and compressive uniaxial strain in [100] changes the tetragonality of the $\mathrm{PbTiO}_{3}$ lattices, which can cause variation in the polarization direction as well as the magnitude. Here, we introduced the polarization direction angle,

$$
\varphi=\tan ^{-1}\left(\delta_{y} / \delta_{x}\right) .
$$

( $\varphi=0^{\circ}$ in $\mathrm{P}[100]$ and $\varphi=45^{\circ}$ in $\left.\mathrm{P}[110]\right)$. Figure 6 shows changes in the polarization distortion per area $\left(\delta^{\prime}\right)$ and direction $(\varphi)$ at the $(1 \times 1)$ surfaces with respect to $\varepsilon_{x x}$. In both terminations, the polarization direction rotates toward [100] under tension and [010] under compression due to the preference of the polarization to the longitudinal direction of rectangular lattices. Although the polarization distortions differ with the layer, their directions are identical in all the layers. While the evolution of $\delta$ with growing tensile strain is similar to that in the case of isotropic tension, the distortion is less suppressed under compression. No critical strain for ferroelectricity is found regardless of the termination layer. 
(a) $\mathrm{TiO}_{2}$ termination
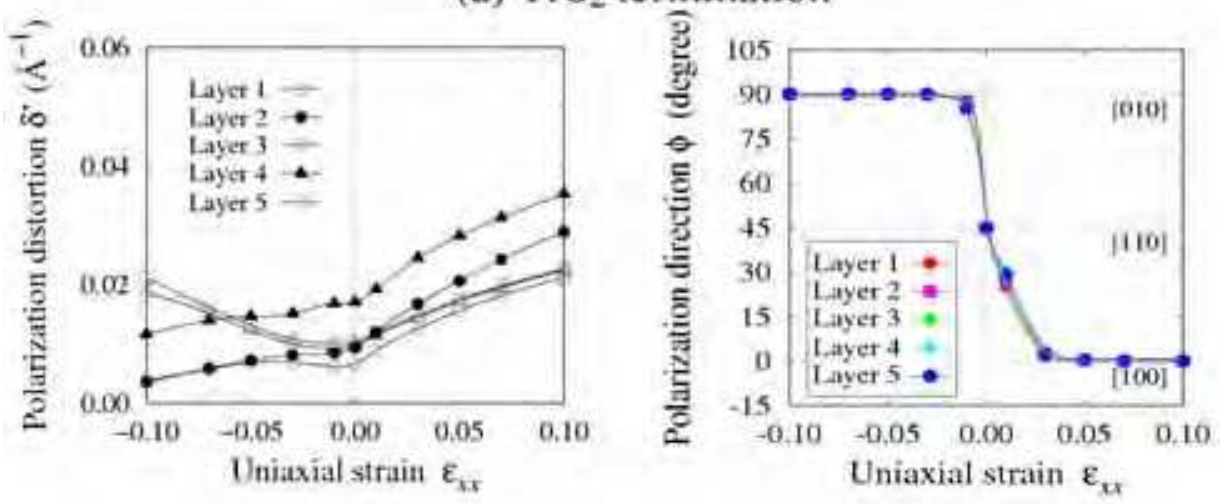

(b) $\mathrm{PbO}$ termination
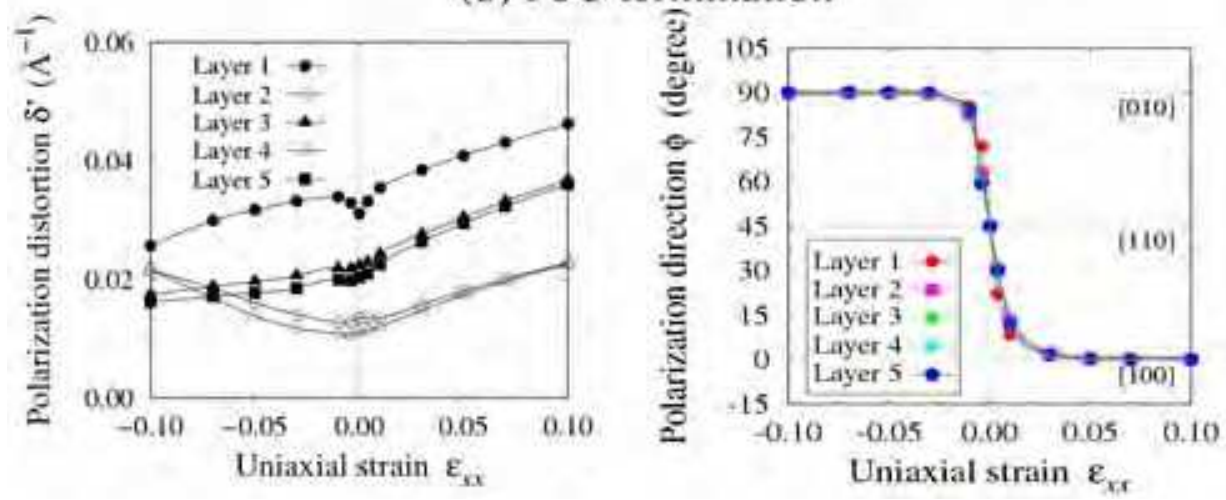

Fig. 6. Polarization distortion per area, $\delta^{\prime}$, and polarization direction angle, $\varphi$, as a function of uniaxial strain, $\varepsilon_{x x}$. (a) $\mathrm{TiO}_{2}$ - and (b) PbO-terminations.

\subsection{Effect of $c(2 \times 2)$ reconstruction on ferroelectricity at surfaces}

For the PbO-terminated surface, there exists the $\mathrm{c}(2 \times 2)$ surface reconstruction incorporated with the rotation of the $\mathrm{TiO}_{4}$ square, which is denoted as the antiferrodistortive (AFD). The atomic displacement of AFD is depicted in Fig. 7.

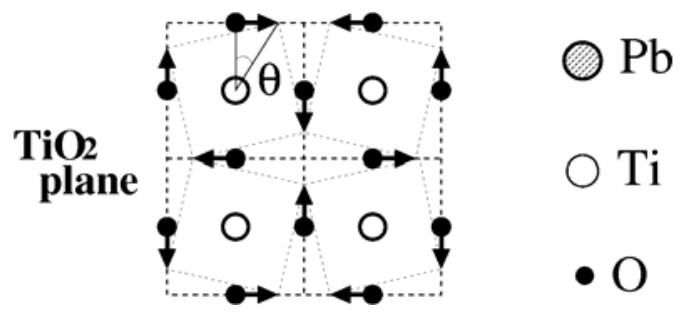

Fig. 7. Schematic illustration explaining the structural parameter of antiferrodistortive (AFD) rotation angle, $\theta$. 
Figure 8 shows the simulation model for the $c(2 \times 2)$ reconstructed surface. The simulation procedure is the same as the $(1 \times 1)$ case in the previous section.
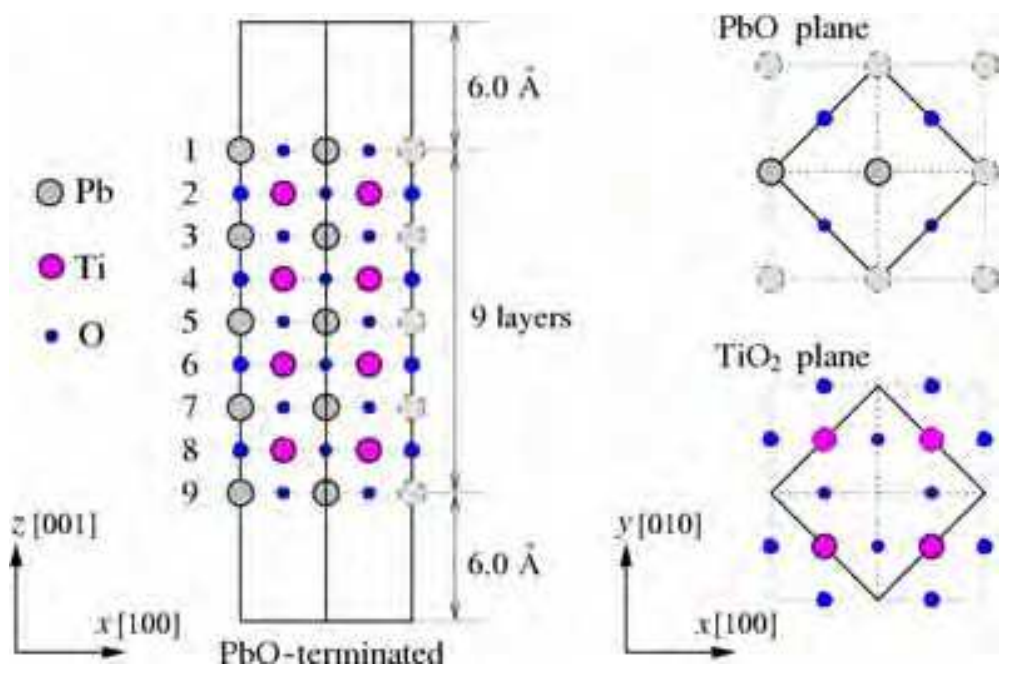

Fig. 8. Simulation models of $\mathrm{PbTiO}_{3}(001)$ surface of the PbO-termination with the $\mathrm{c}(2 \times 2)$ reconstruction. Solid boxes represent simulation cell.

Figure 9 shows atomic structures of the PbO-terminated $c(2 \times 2)$ surface, where FE and AFD coexist, and schematic illustrations of FE and AFD distortions. In this phase, the polarization directions of cells A and B in Fig. 9 are not identical. The polarizations are deviated from their average directions ([100] or [110]) clockwise or anticlockwise, which is clearly due to the contribution of AFD. This phase will be denoted as "FE+AFD (coexisted) phase" hereafter. The energy, the polarization distortion and the AFD rotation in this phase are listed in Table 2. The $\varphi_{\text {ave }}$ is the averaged polarization direction $\left(0^{\circ}\right.$ in $\mathrm{P}[100]$ and $45^{\circ}$ in

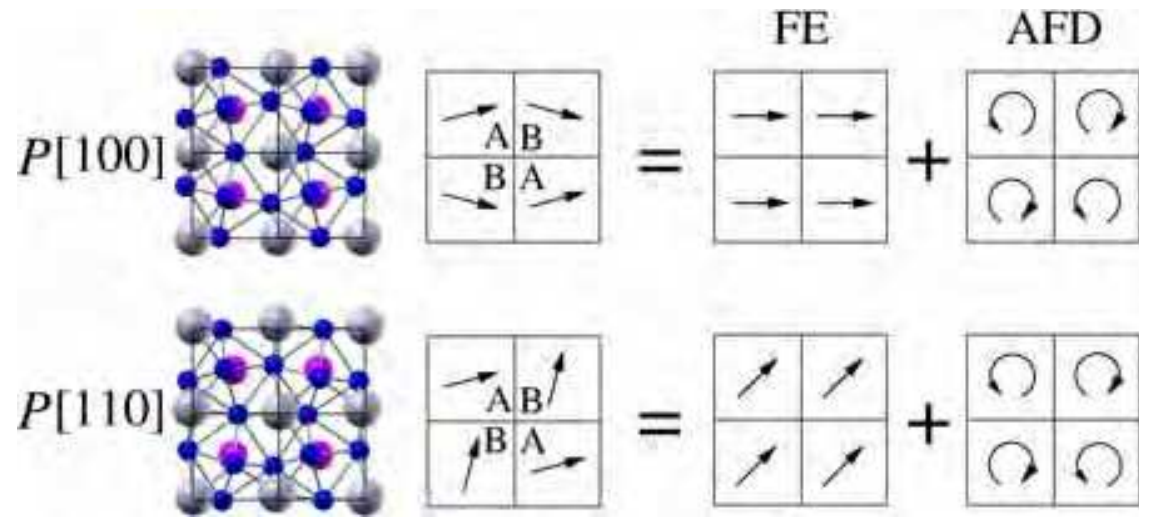

Fig. 9. Atomic structures of $\mathrm{c}(2 \times 2) \mathrm{PbO}$-terminated surfaces. Only the first two layers are shown. Arrows indicate FE and AFD distortions of the first PbO layer and AFD rotation of the adjacent $\mathrm{TiO}_{2}$ layer. 
$\mathrm{P}[110])$, thus $\varphi-\varphi_{\text {ave }}$ denotes the strength of polarization rotation induced by AFD. As for $\theta$, we take the rotation angle of the oxygen squares in the $\mathrm{TiO}_{2}$ planes (see also Fig. 7). An important finding here is that the [110]-polarized structure is energetically the most favored with the nontrivial energy advantage of about $0.075 \mathrm{eV}\left(39.7 \mathrm{~mJ} / \mathrm{m}^{2}\right.$ for one surface side) with respect to the [100]-polarized case. Although the magnitude of the AFD rotation is almost comparable among the two cases, the magnitude of $\varphi-\varphi_{\text {ave }}$ of PbO-layers in P[110] is more than twice larger than that in $\mathrm{P}[100]$.

Figure 10 represents change in the ferroelectric and antiferrodistortive distortions of $\mathrm{P}[110]$ phase of the $\mathrm{c}(2 \times 2) \mathrm{PbO}$-terminated surface. The response of the polarization distortion $\left(\delta^{\prime}\right)$ to the variation of the isotropic strain is similar to that in the $(1 \times 1)$ model. The AFD rotation

\begin{tabular}{|c|c|c|c|}
\hline & Layer & $\begin{array}{c}\mathrm{P}[100] \\
\left(\varphi_{\text {ave }}=0^{\circ}\right)\end{array}$ & $\begin{array}{c}\mathrm{P}[110] \\
\left(\varphi_{\text {ave }}=45^{\circ}\right)\end{array}$ \\
\hline \multirow{4}{*}{$\delta$} & 1 & 12.22 & 10.68 \\
\cline { 2 - 4 } & 2 & 4.18 & 4.36 \\
\cline { 2 - 4 } & 3 & 6.70 & 6.35 \\
\cline { 2 - 4 } & 4 & 3.88 & 4.10 \\
\cline { 2 - 4 } & 5 & 7.69 & 7.29 \\
\hline \multirow{4}{*}{$\varphi_{\text {ave }}$} & 1 & 13.9 & -36.1 \\
\cline { 2 - 4 } & 2 & -6.8 & 1.1 \\
\cline { 2 - 4 } & 3 & -5.4 & 24.7 \\
\cline { 2 - 4 } & 4 & 1.0 & -0.8 \\
\hline \multirow{2}{*}{$\theta$} & 2 & 5.0 & -18.2 \\
\cline { 2 - 4 } & 4 & -0.3 & -0.4 \\
\hline
\end{tabular}

Table 2. Polarization distortion, $\delta$, in percentage of the lattice parameter $\left(a_{0}=3.891 \AA\right)$, polarization direction angle, $\varphi$, and AFD rotation angle, $\theta$, of the cell A in Fig. 9 at PbOterminated $\mathrm{c}(2 \times 2)$ surfaces.
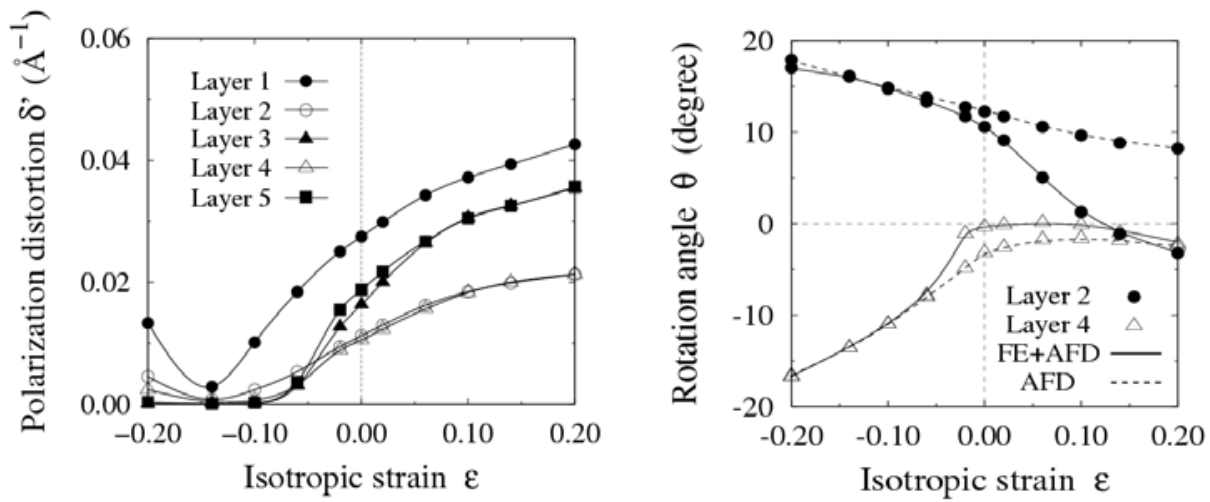

Fig. 10. Polarization distortion per area, $\delta^{\prime}$, and AFD rotation, $\theta$, as a function of isotropic strain, $\varepsilon$, in the $\mathrm{c}(2 \times 2) \mathrm{PbO}$-terminated surface model. Dashed lines in the right figure represent the results of AFD-only phase for comparison. 
has a nature of the opposite trend to FE case (see dashed lines in Fig. 10, which are the results of AFD-only phase). In the FE+AFD coexisted phase, FE and AFD compete with each other, resulting in the AFD rotation being more suppressed by the FE distortion under tension and the FE being more weakened by the AFD under compression.

Figure 11 shows the polarization direction and distortion in the FE+AFD coexisted phase of the $\mathrm{c}(2 \times 2) \mathrm{PbO}$-terminated model. Although the polarization distortion as a function of [100] strain is quite similar to that of $(1 \times 1)$ model, the polarization direction behaves differently. The direction $\varphi$ differs among the layers. The surface layer prefers to have polarization in [110] direction and the adjacent layer is slightly affected. This result suggests that different polarization directions depending on the distance from the surface can be observed at the $\mathrm{PbTiO}_{3}$ surface subjected to a high uniaxial strain.
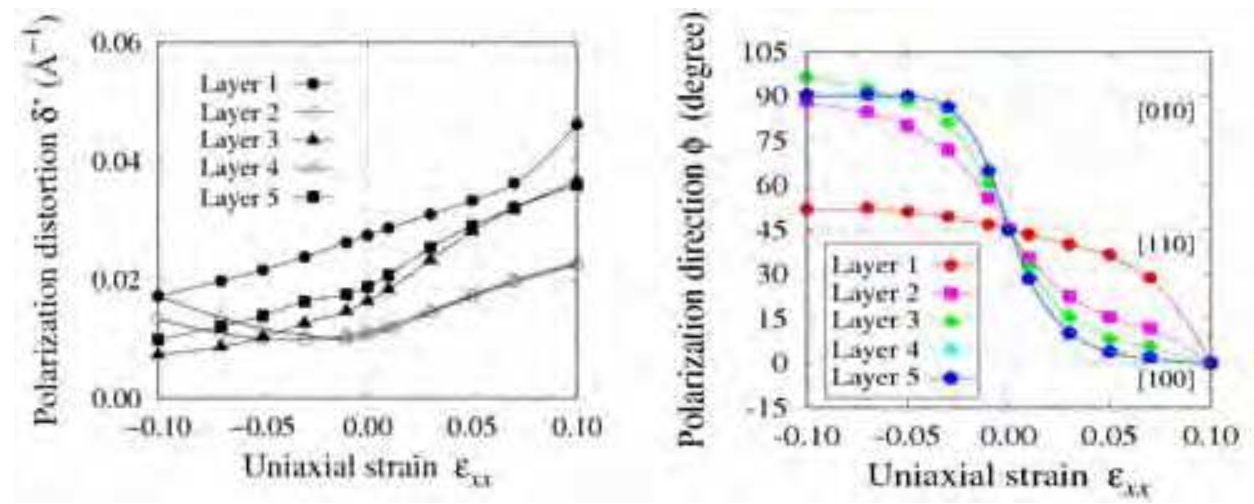

Fig. 11. Polarization distortion per area, $\delta^{\prime}$, and polarization direction angle, $\varphi$, as a function of uniaxial strain, $\varepsilon_{x x}$, in the $\mathrm{c}(2 \times 2)$ PbO-terminated surface model.

Figure 12 depicts change in the atomistic and electronic configurations at the surface layer of the PbO-terminated models. In the $(1 \times 1)$ model, the central oxygen atom is shifted toward a corner $\mathrm{Pb}$ with a strong covalent bond ( $\alpha$ ) and two equivalent weak bonds ( $\beta$ and $\gamma$ ) at $\varepsilon_{x x}=$ 0.0. When a strain is applied in the $x$ direction, the oxygen immediately moves towards either of the equivalent bonds to form two strong bonds; $\alpha$ and $\beta$ under tension or $\alpha$ and $\gamma$ under compression. This bond switching process, which is also found in the $\mathrm{TiO}_{2}-$ terminated surface, explains the polarization rotation very sensitive to the uniaxial strain in the $(1 \times 1)$ periodicity. On the other hand, the $\mathrm{c}(2 \times 2) \mathrm{PbO}$-terminated surface has the different bond structure. Oxygen atoms are shifted in different directions in the lattices A ([010]) and $\mathrm{B}$ ([100]), forming two strong covalent bonds with the nearest $\mathrm{Pb}$ atoms. The bond structure in the lattice A does not immediately change when a small uniaxial tension is applied. At relatively high strain $\left(\varepsilon_{x x}>0.07\right)$ the shift of the oxygen occurs with the break of $\gamma$ bond followed by the construction of $\beta$ bond, consequently forming [100] polarization in both lattices. It is worth noting that the [100]-polarized structure becomes energetically almost identical to the [100]/[010] mixed polarization at a lower strain, $\varepsilon_{x x} \sim 0.05$, which indicates the existence of small energy barrier between those structures for the bond reconstruction of $\beta$ and $\gamma$ bonds. In contrast, the mixed polarization is always more favored than [010] under compression despite the increase in the tetragonal ratio. This is because compression is applied keeping $L_{y}$ (lattice parameter in the $y$ direction) in our calculation, which does not lead to bond breaking required for the reconstruction of polarization structure. 


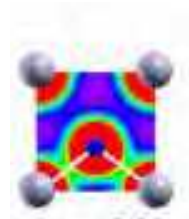

$\varepsilon_{n x}=-0.01$

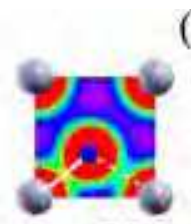

$\varepsilon_{\mathrm{x}}=-0.002$
(1×1) surface

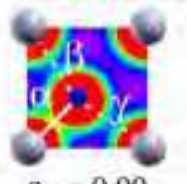

$\varepsilon_{c_{x}}=0.00$

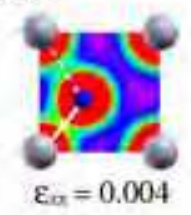

$\varepsilon_{i r}=0.004$

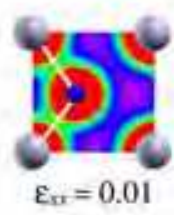

$\varepsilon_{n}=0.01$
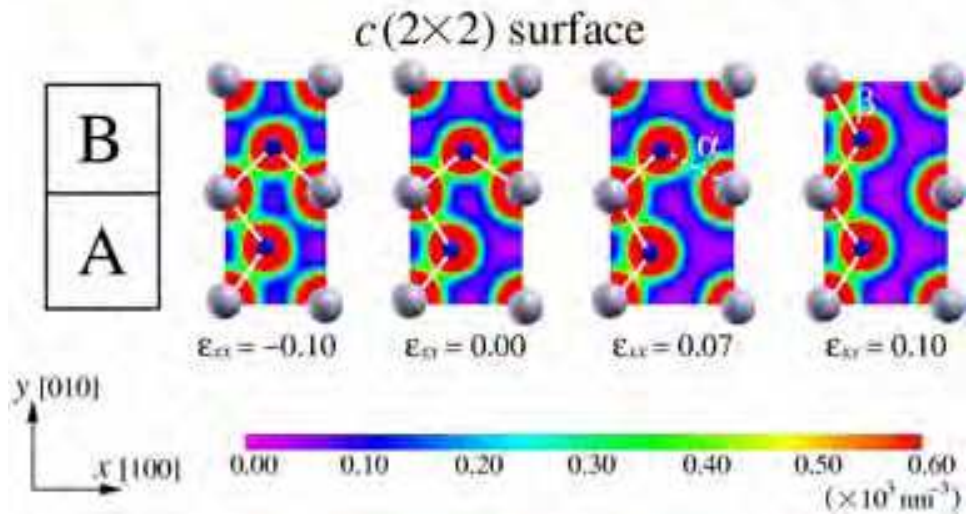

Fig. 12. Change in atomistic and electronic configurations at the first surface layer of the $(1 \times 1)$ and $c(2 \times 2)$ PbO-terminated surface models under uniaxial strain, $\varepsilon_{x x}$.

\section{Ferroelectricity and multi-physics properties in edged nanowires}

\subsection{Simulation models and procedure}

We study $\mathrm{PbTiO}_{3}$ nanowires with edges consisting of (100) and (010) surfaces, where there are two possible terminations of the $\mathrm{PbO}$ and $\mathrm{TiO}_{2}$ atomic layers. Figures $10(\mathrm{a})$ and (b) show the simulation models of the $\mathrm{PbO}$ - and $\mathrm{TiO}_{2}$-terminated nanowires, respectively. Each nanowire has a cross-section in which four perovskite unit cells are arranged in the $x$ and $y$ directions, namely $4 \times 4$ unit cells. Spontaneous polarization aligns along the $z$ direction (the axial direction in the nanowire). Since a three-dimensional periodic boundary condition is applied in the calculations, a vacuum region of $l_{v}=12 \AA$ thickness is introduced in both the $x$ and $y$ directions so that undesirable interaction between the neighboring nanowires is sufficiently avoided. The simulation cell dimensions in the $x, y$ and $z$ directions are initially set to $4 a+l_{v}, 4 a+l_{v}$ and $c$ using the theoretical lattice constants of the tetragonal bulk, $a=3.867$ $\AA$ and $c=4.034 \AA(c / a=1.043)$. The Brillouin zone (BZ) integration is carried out with a $2 \times 2 \times 6 k$-point mesh generated by the Monkhorst-Pack scheme. The condition of $a b$ initio calculations are the same as the case of thin films in the previous section.

As shown in Fig. 10, the unit cells at an edge, on a surface and inside the nanowire are classified into "edge cell", "surface cell" and "inside cell", respectively. The terms "edge bond", "surface bond" and "inside bond" denote the $\mathrm{Pb}-\mathrm{O}$ covalent bonds in the corresponding cells.

To obtain an equilibrium structure, atomic positions and a cell size in the $z$ direction are fully relaxed using the conjugate gradient method until all the Hellmann-Feynman forces

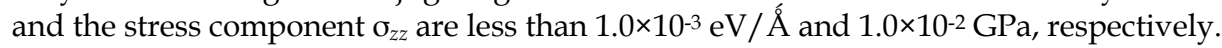


Smaller nanowires with a cross-sections of $1 \times 1,2 \times 2$ and $3 \times 3$ unit cells are investigated in the same manner in order to elucidate the effects of nanowire size and axial strain on ferroelectricity. For tensile simulations, a small axial strain, $\Delta \varepsilon_{z z}$, is applied to the simulation cell step by step. At each strain, internal atoms are fully relaxed under the fixed cell dimensions.

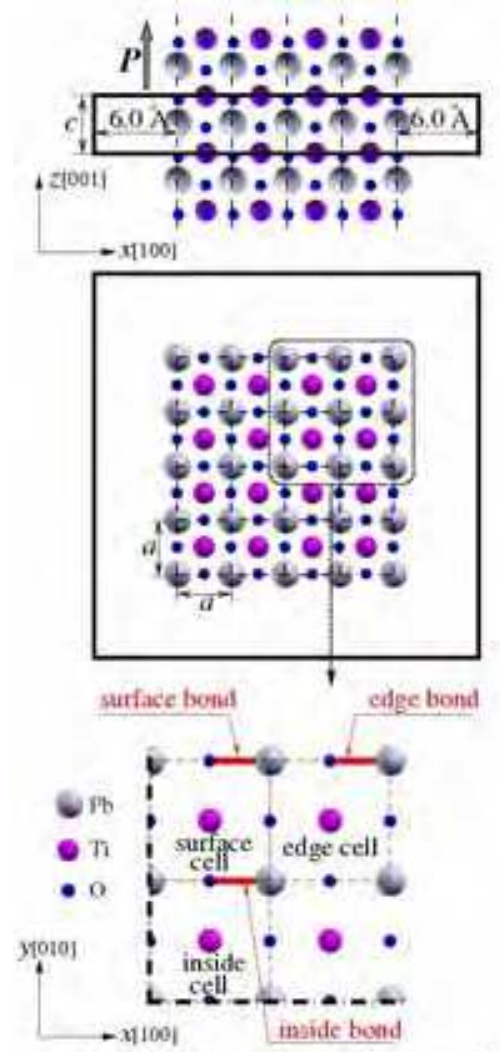

(a) $\mathrm{PbO}$-terminated nanowire
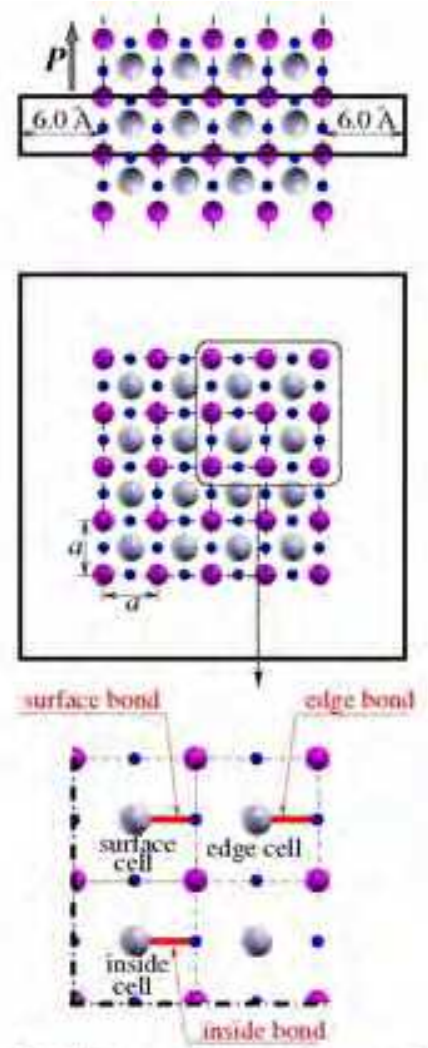

(b) $\mathrm{TiO}_{2}$-terminated nanowire

Fig. 13. Simulation models of (a) $\mathrm{PbO}$-terminated and (b) $\mathrm{TiO}_{2}$-terminated nanowires with a cross-section of $4 \times 4$ unit cells. The solid boxes represent the simulation cells. Polarization $\boldsymbol{P}$ lies along the axial $z$ direction.

\subsection{Ferroelectricity in unstrained nanowires}

The local polarization in the cell, $\mathrm{P}$, is evaluated by

$$
\mathbf{P}=\frac{e}{\Omega_{c}} \sum_{j} w_{j} \mathbf{Z}_{j}^{*} \mathbf{u}_{j}
$$

where $\Omega_{c}, e$ and $\boldsymbol{u}_{j}$ denote the volume of the unit cell, the electron charge and the atomic displacement vector from the ideal lattice site of atom $j$, respectively. Index $j$ runs over all atoms in the unit cell $i$. $\boldsymbol{Z}_{j}^{*}$ is the Born effective charge tensor of the cubic bulk $\mathrm{PbTiO}_{3}$. In this 
study, we use the theoretical values of the Born effective charge tensors calculated by Zhong et al. (Zhong et al., 1994). Weights are set to $w_{\mathrm{Pb}}=1 / 8, w_{\mathrm{Ti}}=1$ and $w_{\mathrm{O}}=1 / 2$ for the Pb-edged cell, and $w_{\mathrm{Pb}}=1, w_{\mathrm{Ti}}=1 / 8$ and $w_{\mathrm{O}}=1 / 2$ for the Ti-edged cell.

Table 3 shows local polarization of the edge, surface and inside cells in the PbO-terminated and $\mathrm{TiO}_{2}$-terminated nanowires with the $4 \times 4$-cells cross-section. The results of the [100]polarized nine-layered $\mathrm{PbTiO}_{3}$ film with the (001) surfaces (Umeno et al., 2006) and the bulk are shown for comparison. For the film, polarization is taken from the magnitude in the unit cell on the surface. In the PbO-terminated nanowire, the edge cell exhibits the highest local polarization. The local polarization of the surface cell, which is second highest, is nearly equal to that of the film. Polarization in the inside cell is also comparable to that of the bulk. On the other hand, little polarization is found in the $\mathrm{TiO}_{2}$-terminated nanowire. More specifically, the inner cell in the nanowire exhibits higher polarization, which is the opposite of the trend found in the PbO-terminated nanowire. Thus, ferroelectricity in the vicinity of the edge is enhanced in the PbO-terminated nanowire, while it is considerably suppressed in the $\mathrm{TiO}_{2}$-terminated nanowire. Note that the $\mathrm{TiO}_{2}$-terminated nanowire shrinks in the $z$ (axial) direction at equilibrium, resulting in considerable suppression of tetragonality, $c / \bar{a}=0.973(c / a=1.043$ for bulk), where $\bar{a}$ denotes the averaged lateral lattice parameter of the wire. It was reported that ferroelectricity in $\mathrm{PbTiO}_{3}$ is suppressed as uniaxial compression is applied along the $c$-axis, meaning the decrease of tetragonality (Shimada et al., 2008). Hence, the tiny magnitude of polarization of the $\mathrm{TiO}_{2}$-termination even in the inside cell is due to not only the effect of $\mathrm{TiO}_{2}$-terminated surface but also the highly reduced tetragonality.

\begin{tabular}{|c|c|c|c|c|c|}
\hline & \multicolumn{3}{|c|}{ Nanowire } & \multirow[b]{2}{*}{ Film } & \multirow[b]{2}{*}{ Bulk } \\
\hline & Edge cell & Surface cell & Inside cell & & \\
\hline $\mathrm{PbO}$ termination & 103.2 & 96.5 & 81.0 & 93.8 & 85.8 \\
\hline $\mathrm{TiO}_{2}$ termination & 0.55 & 0.70 & 1.61 & 47.0 & 85.8 \\
\hline
\end{tabular}

Table 3. Local polarization evaluated via Eq. (10), $P$ (in $\left.\mu \mathrm{C} / \mathrm{cm}^{2}\right)$, of the edge, surface, and inside cells in the $\mathrm{PbO}$-terminated and $\mathrm{TiO}_{2}$-terminated nanowires with a $4 \times 4$-cell cross section. The results for [100]-polarized film with (001) surfaces (Umeno et al., 2006) and the bulk are shown for comparison.

It was both theoretically (Cohen, 1992) and experimentally (Kuroiwa et al., 2001) revealed that the prominent ferroelectricity and large lattice tetragonality in $\mathrm{PbTiO}_{3}$ stems from the formation of the strong covalent $\mathrm{Pb}-\mathrm{O}$ bond through the hybridization of the $\mathrm{Pb} 6 \mathrm{~s}$ and $\mathrm{O} 2 \mathrm{p}$ orbitals, unlike $\mathrm{BaTiO}_{3}$ where the Ba-O interaction is almost ionic. In addition, the $\mathrm{Pb}-\mathrm{O}$ bond often plays a significant role in determining characteristic structures, such as a complex $c(2 \times 2)$ surface reconstruction (Shimada et al., 2006) and an atomically sharp $90^{\circ}$ domain wall structure (Shimada et al., 2008). Thus, the $\mathrm{Pb}-\mathrm{O}$ bond characterizes ferroelectric instability in $\mathrm{PbTiO}_{3}$. Table 4 lists the bond length, $d$, and minimum charge density, $\rho_{\text {min, }}$ of the edge, surface and inside $\mathrm{Pb}-\mathrm{O}$ bonds in both the $\mathrm{PbO}$ - and $\mathrm{TiO}_{2}$-terminated nanowires (see Fig. 10). The minimum charge density is calculated on a line between the bonded $\mathrm{Pb}$ and $\mathrm{O}$ atoms. For comparison, we also show those of the bonds in the bulk and in the film as mentioned above, whose sites correspond to the surface and inside bonds in the nanowire. In the PbO-terminated nanowire, the bond length is in order, $d_{\text {edge }}<d_{\text {surface }}\left(<d_{\text {film }}\right)<d_{\text {inside }}$ $\left(\approx d_{\text {bulk }}\right)$, and the minimum charge density is in order, $\rho_{\min }{ }^{\text {edge }}>\rho_{\min }$ surface $>\left(\rho_{\text {min }}\right.$ film $\left.>\right)$ $\rho_{\text {min }}$ inside $\left(\approx \rho_{\text {min }}\right.$ bulk $)$. These findings indicate that a highly strengthened covalent bond is 
formed at the edge. On the other hand, $d$ and $\rho_{\text {min }}$ are longer and lower than those of the bulk in the $\mathrm{TiO}_{2}$-terminated nanowire, suggesting that the $\mathrm{Pb}-\mathrm{O}$ bond is weakened. Therefore, the large (respectively, small) ferroelectric distortion in the edge cell arises from the strengthened (respectively, weakened) covalent $\mathrm{Pb}-\mathrm{O}$ bond in the $\mathrm{PbO}$ - (respectively, $\left.\mathrm{TiO}_{2-}\right)$ terminated nanowire.

\begin{tabular}{|c|c|c|c|c|c|}
\hline \multirow{2}{*}{} & \multicolumn{3}{|c|}{ Nanowire } & \multirow{2}{*}{ Film } & Bulk \\
\cline { 2 - 4 } & Edge bond & Surface bond & Inside bond & \\
\hline$d$ & 2.243 & 2.299 & 2.464 & 2.386 & 2.506 \\
\hline$\rho_{\text {min }}$ & 0.534 & 0.473 & 0.332 & 0.392 & 0.312 \\
\hline \multicolumn{7}{|c|}{$\mathrm{TiO}_{2}$ termination } \\
\hline$\rho_{\text {min }}$ & 2.675 & 2.722 & 2.697 & 2.631 & 2.506 \\
\hline
\end{tabular}

Table 4. Bond length $d$ (in $\AA$ ), and minimum charge density $\rho_{\min }\left(\right.$ in $\left.\AA^{-3}\right)$, of the $\mathrm{Pb}-\mathrm{O}$ covalent bond in the edge, surface, and inside cells. The corresponding $\mathrm{Pb}-\mathrm{O}$ bonds in the bulk and on the (001) surface of the nine-layered film (Umeno et al., 2006) are shown for comparison.

Figure 11 depicts the difference in site-by-site minimum charge density between the corresponding site of the bulk and those in the $\mathrm{PbO}$ - and $\mathrm{TiO}_{2}$-terminated nanowires. In the $\mathrm{PbO}$-terminated nanowire, the charge density at the edge and surface $\mathrm{Pb}-\mathrm{O}$ bonds increases, while that of the Ti-O bond decreases. This indicates that the electrons transfer from the Ti$\mathrm{O}$ site to the $\mathrm{Pb}-\mathrm{O}$ site. Because the coordination number of the $\mathrm{Pb}$ atom at the edge is smaller than that of the bulk, the number of electrons contributing to the bond increases in the $\mathrm{PbO}$-terminated nanowire. In fact, the $\mathrm{Pb}$ atoms associated with the edge, surface and
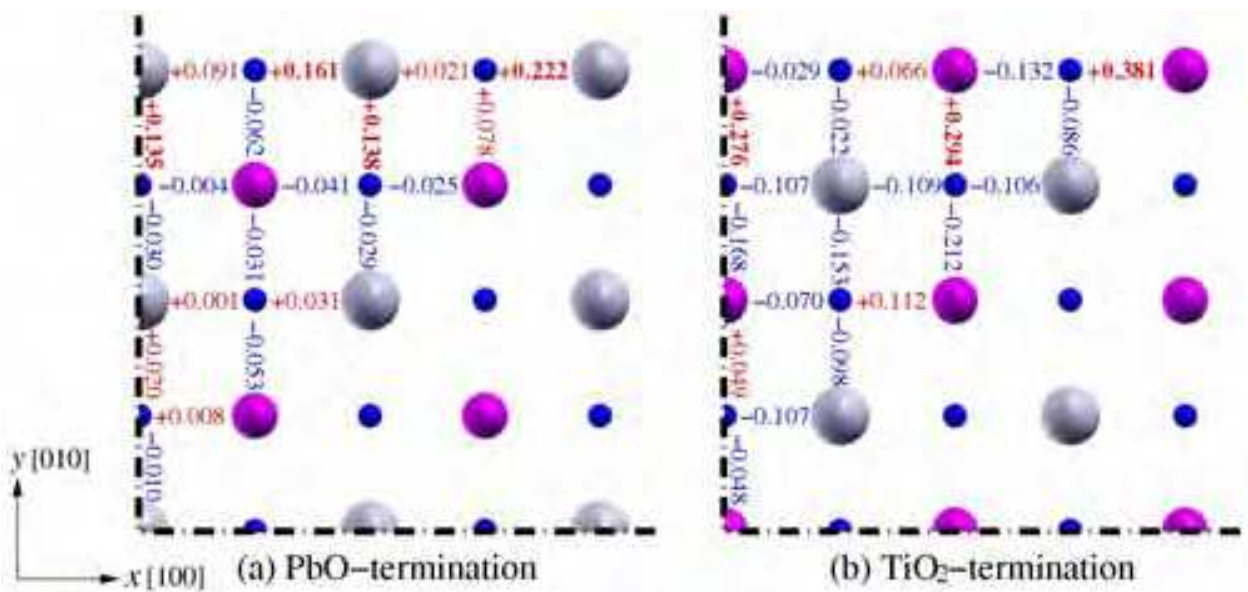

Fig. 14. Difference in site-by-site minimum charge density from the corresponding site in the bulk in (a) PbO-terminated and (b) $\mathrm{TiO}_{2}$-terminated nanowires with a cross-section of $4 \times 4$ unit cells (in $\AA^{-3}$ ). Only the top right quarter of the cross-section is shown by symmetry. The number in red and blue indicate the increase and decrease, respectively. 
inside bonds form 2, 3 and $4 \mathrm{~Pb}-\mathrm{O}$ bonds, respectively, which correspond well to the order in the minimum charge density although the relationship is not simply linear (see Table 4). Thus, the electron concentration on the edge bond due to the reduced coordination number results in locally enhanced ferroelectricity. On the other hand, charge density increases at the Ti-O bond, especially, at the edge in the $\mathrm{TiO}_{2}$-terminated nanowire, while it decreases at the $\mathrm{Pb}-\mathrm{O}$ bond. The enhancement of the $\mathrm{Ti}-\mathrm{O}$ bond can be explained in the same manner as that of the $\mathrm{Pb}-\mathrm{O}$ bond in the PbO-terminated nanowire. As a result of the charge redistribution from the $\mathrm{Pb}-\mathrm{O}$ site to $\mathrm{Ti}-\mathrm{O}$ site in the nanowire, the covalent $\mathrm{Pb}-\mathrm{O}$ bond is relatively weakened, leading to strong suppression of ferroelectricity. Thus, ferroelectricity in the $\mathrm{PbTiO}_{3}$ nanowire depends significantly on the terminations.

\subsection{Finite size effect of nanowires on ferroelectricity}

Smaller nanowires with a cross-sections of $1 \times 1,2 \times 2$ and $3 \times 3$ unit cells are investigated in the same manner in order to elucidate the effects of nanowire size on ferroelectricity. Table 5 lists the averaged lateral lattice parameter and the axial lattice parameter, $\bar{a}$ and $c$, and the averaged polarization, $P$, in both the $\mathrm{PbO}$ - and $\mathrm{TiO}_{2}$-terminated nanowires as a function of cross-sectional size. As the size of the PbO-terminated nanowire decreases, both the lateral and axial lattice parameters shrink. However, the tetragonality of lattice, $c / \bar{a}$, which plays an important role in stabilizing ferroelectric distortion, increases in the smaller nanowire. Surprisingly, even the smallest $\mathrm{PbO}$-terminated nanowire with a $1 \times 1$ unit cell size exhibits spontaneous polarization, which has the highest magnitude among the nanowires and bulk. This is because the ratio of the edge structure that enhances ferroelectricity with respect to the entire wire volume becomes more dominant with decreasing nanowire size. The magnitude of polarization approaches that of the bulk as the size increases. On the other hand, the lattice parameters of the smaller $\mathrm{TiO}_{2}$-terminated nanowire also shrink, but the tetragonality decreases because the axial lattice parameter is highly suppressed. Moreover, nanowires smaller than $4 \times 4$ cells in cross-section, which corresponds to a diameter of about $17 \AA$, do not exhibit spontaneous polarization, indicating that there is the critical size for ferroelectricity. This indicates that critical size for ferroelectricity in perovskite nanowires is much sensitive to surface terminations.

\begin{tabular}{|c|c|c|c|c|c|}
\hline Nanowire size & $1 \times 1$ & $2 \times 2$ & $3 \times 3$ & $4 \times 4$ & Bulk \\
\hline \multicolumn{6}{|c|}{ PbO termination } \\
\hline $\bar{a}$ & 3.60 & 3.64 & 3.70 & 3.73 & 3.87 \\
\hline$c$ & 3.92 & 3.97 & 3.99 & 4.01 & 4.03 \\
\hline$c / \bar{a}$ & 1.088 & 1.090 & 1.081 & 1.074 & 1.043 \\
\hline$P$ & 103.7 & 99.9 & 95.7 & 94.3 & 85.8 \\
\hline $\bar{\sigma}$ & $\mathrm{TiO}_{2}$ termination \\
\hline \multicolumn{7}{|c|}{} & 3.81 & 3.84 & 3.85 & 3.87 \\
\hline$c$ & 3.77 & 3.57 & 3.67 & 3.74 & 4.03 \\
\hline$c / \bar{a}$ & 3.39 & 0.935 & 0.956 & 0.973 & 1.043 \\
\hline$P$ & 0.900 & 0.00 & 0.00 & 0.89 & 85.8 \\
\hline
\end{tabular}

Table 5. Averaged lateral and axial lattice parameters, $\bar{a}$ and $c$ (in $\AA$ ), and averaged polarization $P$ (in $\left.\mu \mathrm{C} / \mathrm{cm}^{2}\right)$, in both the $\mathrm{PbO}$-terminated and $\mathrm{TiO}_{2}$-terminated nanowires as a function of cross-sectional size. 


\subsection{Role of axial tensile strain}

Figure 12 plots the averaged polarization, $\mathrm{P}$, in the $\mathrm{PbO}$-terminated and $\mathrm{TiO}_{2}$-terminated nanowires as a function of the tensile strain, $\varepsilon_{z z}$. Averaged polarization increases almost linearly with respect to tensile strain for all the PbO-terminated nanowires. This suggests that the axial tensile strain enhances the ferroelectricity. For the $\mathrm{TiO}_{2}$-terminated nanowires which are initially paraelectric, ferroelectricity appears under axial tension. The critical tensile strain to recover ferroelectricity is listed in Table 6. Smaller nanowires require larger critical strain because the $\mathrm{TiO}_{2}$-terminated edge structure, which suppresses ferroelectricity, strongly affects thinner nanowires. After the critical strain, averaged polarization increases smoothly with tensile strain.

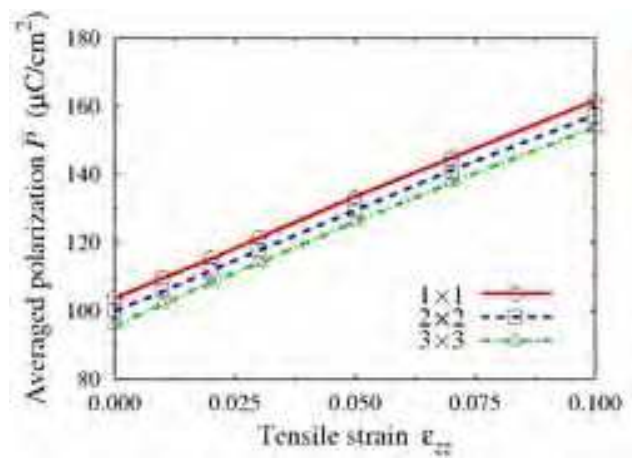

(a) PbO-terminated nanowires

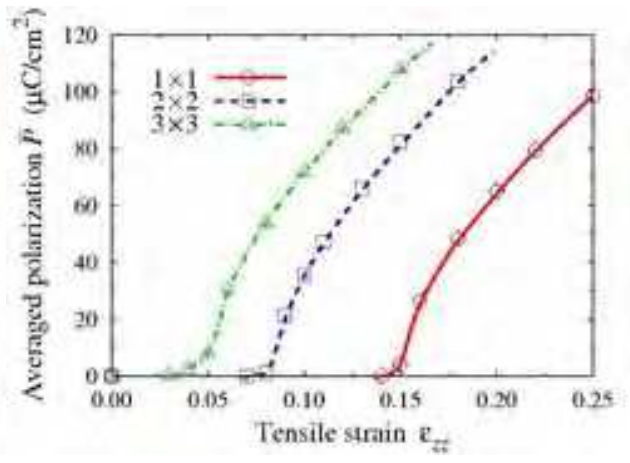

(b) $\mathrm{TiO}_{2}$-terminated nanowires

Fig. 15. Average polarization $P$ in (a) $\mathrm{PbO}$-terminated and (b) $\mathrm{TiO}_{2}$-terminated nanowires with a cross-section of $1 \times 1,2 \times 2$ and $3 \times 3$ unit cells as a function of tensile strain $\varepsilon_{z z}$.

\begin{tabular}{|c|c|c|c|}
\hline Nanowire size & $1 \times 1$ & $2 \times 2$ & $3 \times 3$ \\
\hline$\varepsilon_{c}$ & 0.15 & 0.08 & 0.04 \\
\hline
\end{tabular}

Table 6. Critical tensile strain to recover ferroelectricity $\varepsilon_{c}$ as a function of the $\mathrm{TiO}_{2-}$ terminated nanowire size.

Figure 13 shows the change in atomistic and electronic configurations on the $\mathrm{PbO}(010)$ planes in the $\mathrm{PbO}$-terminated and $\mathrm{TiO}_{2}$-terminated nanowires with a cross-section of $3 \times 3$ unit cells under axial tension. At equilibrium in the $\mathrm{PbO}$-terminated nanowire, $\mathrm{O}$ atoms are displaced in the $-z$ direction from their ideal lattice sites and the covalent $\mathrm{Pb}-\mathrm{O}$ bonds emphasized by white lines are formed, indicating ferroelectric distortion. During axial tension, all the $\mathrm{Pb}-\mathrm{O}$ covalent bonds are sustained and the charge density at these sites increases. On the other hand, the upper and lower $\mathrm{Pb}-\mathrm{O}$ bonds are equivalent to each other and there is no ferroelectric distortion in the unstrained $\mathrm{TiO}_{2}$-terminated nanowire. After the critical strain of $\varepsilon_{c}=0.04$, the upper bond is strengthened while the lower one is weakened for all the $\mathrm{PbO}$ planes accompanying the upward displacement of the $\mathrm{Pb}$ atom. As a result, the nanowire undergoes a paraelectric-to-ferroelectric (PE-FE) phase transition.

For more detailed discussion, we define the "edge", "surface" and "inside" bonds in the $3 \times 3$ nanowire in the same manner as in the $4 \times 4$ nanowires (see Fig. 10). Figure 14 plots the minimum charge density, $\rho_{\min }$, at the edge, surface and inside bonds in the PbO-terminated and $\mathrm{TiO}_{2}$-terminated nanowires with a cross-section of $3 \times 3$ cells as a function of axial tensile 
(a) Pho-terminated nanowire

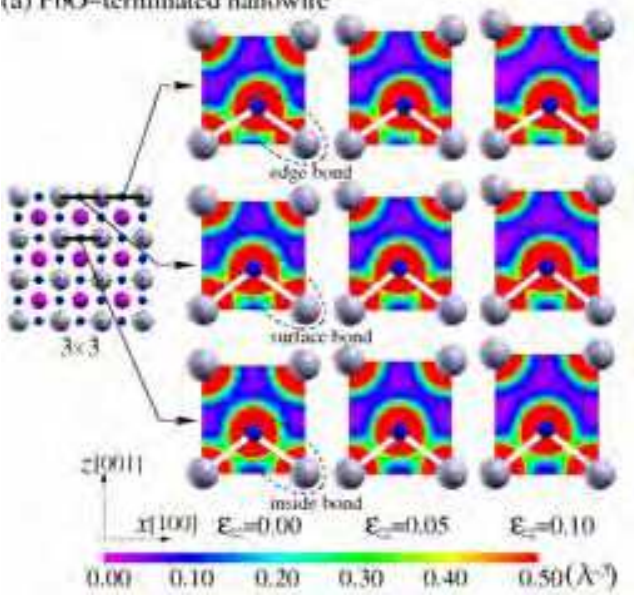

(b) $\mathrm{TiO}$-terminated nanowire

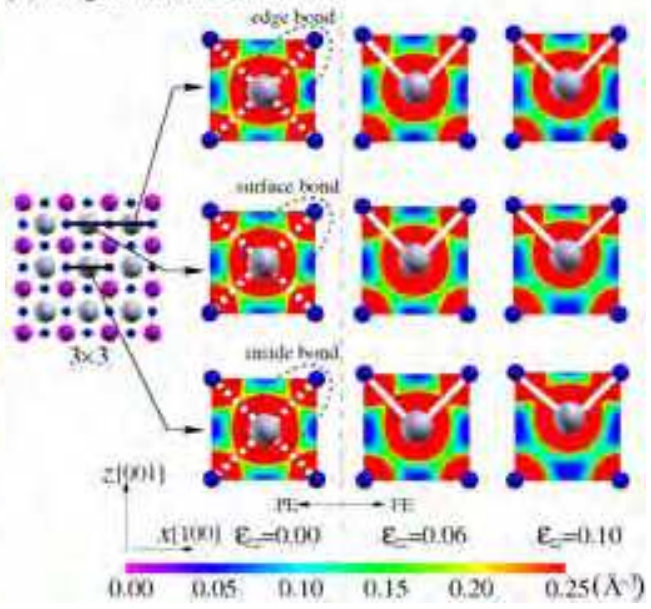

Fig. 16. Change in atomic structures and charge density distribution on the $\mathrm{PbO}(010)$ planes in (a) PbO-terminated and (b) $\mathrm{TiO}_{2}$-terminated nanowires with a cross-section of $3 \times 3$ unit cells under axial tensile strain $\varepsilon_{z z}$. The gray and blue spheres indicate $\mathrm{Pb}$ and $\mathrm{O}$ atoms, respectively. The covalent $\mathrm{Pb}-\mathrm{O}$ bonds are shown by white lines.

strain, $\varepsilon_{z z}$. The minimum charge density at each bond increases monotonically with respect to the tensile strain in the PbO-terminated nanowire. This corresponds well to the linear increase in the averaged polarization. Under high axial tension, the edge bond keeps the highest charge density of the three. In the $\mathrm{TiO}_{2}$-terminated nanowire, the minimum charge density decreases before the critical strain. This indicates that the equivalent bonds at the upper and lower sites of the $\mathrm{PbO}$ plane are uniformly stretched because the structure is paraelectric (symmetric in the $z$ direction). At the critical strain, the trend for all the bonds concurrently changes from decreasing to increasing. This indicates that ferroelectricity
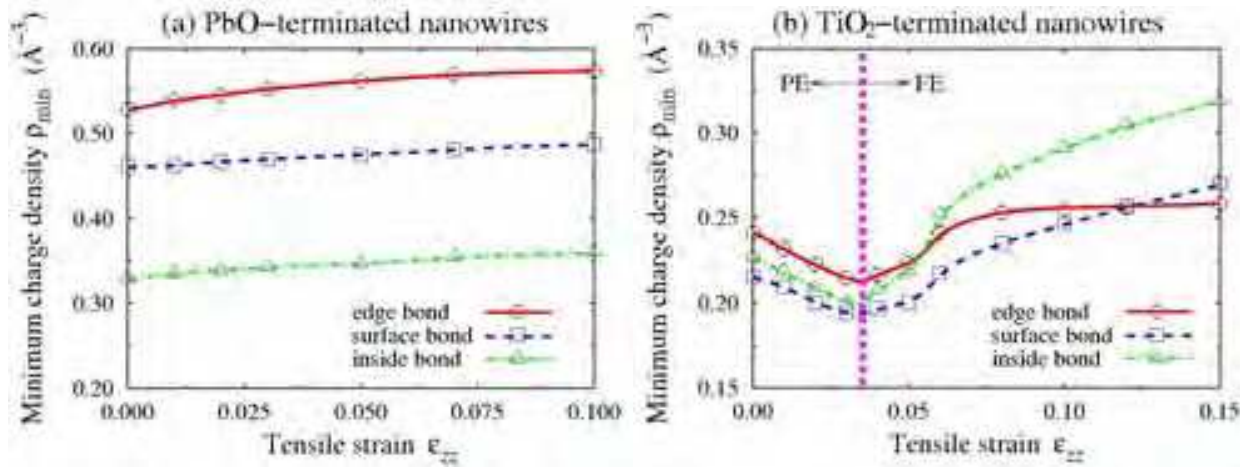

Fig. 17. Minimum charge density $\rho_{\min }$ at the edge, surface and inside bonds (see also Fig. 11) in (a) PbO-terminated and (b) $\mathrm{TiO}_{2}$-terminated nanowires with a cross-section of $3 \times 3$ unit cells as a function of axial tensile strain $\varepsilon_{z z}$. The dashed line indicates the critical strain of the PE-FE phase transition. 
appears in all the sites of the nanowire at the same strain. After that, all the upper bonds are partially strengthened. Note that the increase in charge density at the edge bond is somewhat moderate, which leads to the suppression of ferroelectricity at the edge with respect to the other sites.

\section{Conclusion}

This chapter highlights the prominent ferroelectricity in nanowires focusing on their surface and edge structures from the atomistic and electronic points of view by means of firstprinciples (ab initio) density functional theory calculations. In addition, the coupling effect between the mechanical deformation and ferroelectrictricity, namely, the multi-physics property is reviewed, as well.

At a surface, ferroelectricity, which depends on the terminations, is suppressed by $\mathrm{TiO}_{2}-$ termination and is enhanced by $\mathrm{PbO}$-termination. We demonstrate the strong influence of lateral isotropic strain on the FE and AFD distortions. The ferroelectricity is enhanced by lateral tensile strain while it is suppressed by compression. At the $\mathrm{TiO}_{2}$-terminated surface, ferroelectricity disappears when the compressive strain reaches a critical value. On the other hand, in the $\mathrm{PbO}$ termination, ferroelectricity is not suppressed by the surface and thus polarization distortion does not vanish under high compressive strain. These results indicate the surface FE and AFD distortions can be tuned by applied load. Furthermore we examine the effect of uniaxial [100] strain and find the rotation of ferroelectric polarization direction, which prefers to be along the longitudinal direction of rectangular lattices. While the response is sharp at the $(1 \times 1)$ surfaces, the surface layer exhibit [110]-oriented FE polarization up to relatively high tensile/compressive strains at the PbO-terminated $\mathrm{c}(2 \times 2)$ surface. In addition, polarization direction differs among the layers (Shimada et al., 2006).

For the nanowires with atomically sharp edges consisting of (100) and (010) surfaces, the ferroelectric distortions at the edge of the PbO-terminated nanowire are enhanced, while they are entirely suppressed in the $\mathrm{TiO}_{2}$-terminated nanowire. A precise investigation from the electronic state has shown that ferroelectricity is enhanced as a result of the formation of strong $\mathrm{Pb}-\mathrm{O}$ covalent bonds at the edge of the $\mathrm{PbO}$-terminated nanowire. This arises from the increase of electrons contributing to the bond due to the relative decrease in the coordination number of the $\mathrm{Pb}$ atom. In the $\mathrm{TiO}_{2}$-terminated nanowire, the $\mathrm{Pb}-\mathrm{O}$ bonds are weakened by the charge-transfer from the $\mathrm{Pb}-\mathrm{O}$ site to the Ti-O site. Surprisingly, the smallest PbO-terminated nanowire with a cross-section of only one unit cell exhibits spontaneous polarization, which has a higher magnitude than the bulk. By contrast, the $\mathrm{TiO}_{2}$-terminated nanowires with a cross-section smaller than $4 \times 4$ cells (about $17 \AA$ in a diameter) cannot sustain ferroelectricity, indicating the existence of critical size. Spontaneous polarization in the PbO-terminated nanowires increases almost proportionally to the applied tensile strain accompanying the homogeneous enhancement of the $\mathrm{Pb}-\mathrm{O}$ bond. In the $\mathrm{TiO}_{2}$-terminated nanowires, which are initially paraelectric, the ferroelectricity emerges under axial tension. A larger critical strain is required for smaller nanowires, where the suppressing effect of the edge on ferroelectricity becomes predominant (Shimada et al., 2009).

There are further challenging topics on the ferroelectric nanowires remained. For example, a magnetic-like vortex ferroelectric structure was predicted in a nanowire by macroscopic conventional theory, but such vortex structure has not yet been investigated from the atomistic points of view. Besides, a recent experimental investigation revealed that the 
characteristic domain structure is incorporated with the nanowires, and the domain period is highly depends on the size of nanowires. It is expected that the atomic and electronic structure at the junction of atomically sharp edge (or surface) in nanowires and the domain wall significantly differs from those of bulk. It would be also interesting to study the influence of the junction in the nanowires. These issues will be addressed in the near future.

\section{Acknowledgements}

We acknowledge financial support by a Grant-in-Aid for Scientific Research (S) (Grant No. 21226005) and a Grant-in-Aid for Young Scientists (B) (Grant No. 21760073), of the Japan Society of the Promotion of Science (JSPS). The authors would also like to acknowledge fruitful discussions and kind support with Mr. Shogo Tomoda.

\section{References}

Spanier, J.E. ; Kolpak, A.M. ; Urban, J.J. ; Grinberg, I. ; Ouyang, L. ; Yun, W.S. ; Rappe, A.M. \& Park, H. (2006). Ferroelectric phase transition in individual single-crystalline $\mathrm{BaTiO}_{3}$ nanowires, Nano Letters, Vol. 6, No. 4 (April 2006) pp. 735-739, ISSN 15306984

Scott, J.F. (2000). Ferroelectric Memories, Springer, Berlin

Gu, H. ; Hu, Y. ; You, J. ; Hu, Z. ; Yuan, Y. \& Zhang, T. (2007). Characterization of single crystalline $\mathrm{PbTiO}_{3}$ nanowire growth via surfactant-free hydrothermal method, Journal of Applied Physics, Vol. 101, No. 2 (January 2007) 024319, ISSN 0021-8979

Yamashita, Y. ; Mukai, K. ; Yoshinobu, J. ; Lippmaa, M. ; Kinoshita, T. \& Kawasaki, M. (2002). Chemical nature of nanostructures of $\mathrm{La}_{0.6} \mathrm{Sr}_{0.4} \mathrm{MnO}_{3}$ on $\mathrm{SrTiO}_{3}$ (100), Surface Science, Vol. 514, No. 1-3 (August 2002) pp. 54-59, ISSN 0039-6028

Cho, G.-B. ; Yamamoto, M. \& Endo, Y. (2004). Surface features of self-organized $\mathrm{SrTiO}_{3}(001)$ substrates inclined in [100] and [110] directions, Thin Solid Films, Vol. 464-465, (October 2004) pp. 80-84, ISSN 0040-6090

Kotomin, E.A.; Heifets, E.; Dorfman, S. ; Fuks, D.; Gordon, A. \& Maier, J. (2004). Comparative study of polar perovskite surfaces, Surface Science, Vol. 566-568, (September 2004) pp. 231-235, ISSN 0039-6028

Chu, M.W. ; Szafraniak, I. ; Scholz, R. ; Harnagea, C. ; Hesse, D. ; Alexe, M. \& Gosele, U. (2004). Impact of misfit dislocations on the polarization instability of epitaxial nanostructured ferroelectric perovskites, Nature Materials, Vol. 3, No. 2 (February 2004) pp. 87-90, ISSN 1476-1122

Chakrabarti, N.B. ; Ray, S.K. \& Maiti, C.M. (2001). Strained Silicon Heterostructures : Materials and Devices, Inspec/Iee, England

Umeno, Y. \& Kitamura, T. (2002). Ab initio simulation on ideal shear strength of silicon, Materials Science and Engineering B, Vol. 88, No. 1 (January 2002) pp. 79-84, ISSN 0921-5107

Hohenberg, P. \& Kohn, W. (1964). Inhomogeneous electron gas, Pysical Review, Vol. 136, No. 3B, B864, ISSN 0163-1829

Kohn, W. \& Sham, L. (1965). Self-consistent equations including exchange and correlation effects, Physical Review, Vol. 140, No. 4A, 1133, ISSN 0031-899X

Blochl, P.E. (1994). Projector augmented-wave method, Physical Review B, Vol. 50, No. 24 (December 1994) pp. 17953-17979, ISSN 0163-1829 
Kresse, G. \& Hafner, J. (1993). Ab initio molecular dynamics for liquid metals, Physical Review B, Vol. 47, No. 1 (January 1993) pp. 558-561, ISSN 0163-1829

Kresse, G. \& Furthmuller, J. (1996). Efficient iterative schemes for ab initio total-energy calculations using a plane-wave basis set, Physical Review B, Vol. 54, No. 16 (October 1996) pp. 11169-11186, ISSN 0163-1829

Ceperley, D.M. \& Alder, B.J. (1980). Ground state of electron gas by a stochastic method, Physical Review Letters, Vol. 45, No. 7, pp. 566-569, ISSN 0031-9007

Monkhorst, H.J. \& Pack, J.D. (1976). Special points for Brillouin-zone integrations, Physical Review B, Vol. 45, No. 12, pp. 5188-5192, ISSN 0163-1829

Zhong, W. ; King-Smith, R.D. \& Vanderbilt, D. (1994). Giant LO-TO splitting in perovskite ferroelectrics, Physical Review Letters, Vol. 72, No. 22 (May 1994) pp. 3618-3621, ISSN 0031-9007

Umeno, Y.; Shimada, T. ; Kitamura, T. \& Elsaesser, C. (2006). Ab initio density functional theory study of strain effects on ferroelectricity at $\mathrm{PbTiO}_{3}$ surfaces, Physical Review B, Vol. 74, No. 17 (November 2006) 174111, ISSN 1098-0121

Shimada, T. ; Wakahara, K. ; Umeno, Y. \& Kitamura, T. (2008). Shell model potential for $\mathrm{PbTiO}_{3}$ and its applicability to surfaces and domain walls, Journal of Physics: Condensed Matter, Vol. 20, No. 32 (Augst 2008) 325225, ISSN 0953-8984

Cohen, R.E. (1992). Origin of ferroelectricity in perovskite oxides, Nature, Vol. 358 (July 1992) pp. 136-138, ISSN 0028-0836

Kuroiwa, Y. ; Aoyagi, S. ; Sawada, A. ; Harada, J. ; Nishibori, E. ; Tanaka, M. \& Sakata, M. (2001). Evidence for $\mathrm{Pb}-\mathrm{O}$ covalency in tetragonal $\mathrm{PbTiO}_{3}$, Physical Review Letters, Vol. 87, No. 21 (November 2001) 217601, ISSN 0031-9007

Shimada, T. ; Umeno, Y. \& Kitamura, T. (2008). Ab initio study of stress-induced domain switching in $\mathrm{PbTiO}_{3}$, Physical Review B, Vol. 77, No. 9 (March 2008) 094105, ISSN 1098-0121

Shimada, T. ; Tomoda, S. \& Kitamura, T. (2009). Ab initio study of ferroelectricity in edged $\mathrm{PbTiO}_{3}$ nanowires under axial tension, Physical Review B, Vol. 79, No. 2 (January 2009) 024102, ISSN 1098-0121 


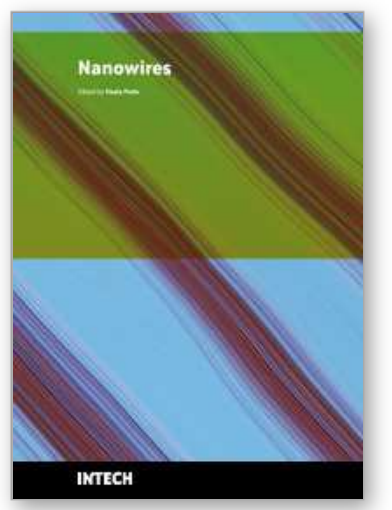

\author{
Nanowires \\ Edited by Paola Prete
}

ISBN 978-953-7619-79-4

Hard cover, 414 pages

Publisher InTech

Published online 01, February, 2010

Published in print edition February, 2010

This volume is intended to orient the reader in the fast developing field of semiconductor nanowires, by providing a series of self-contained monographs focusing on various nanowire-related topics. Each monograph serves as a short review of previous results in the literature and description of methods used in the field, as well as a summary of the authors recent achievements on the subject. Each report provides a brief sketch of the historical background behind, the physical and/or chemical principles underlying a specific nanowire fabrication/characterization technique, or the experimental/theoretical methods used to study a given nanowire property or device. Despite the diverse topics covered, the volume does appear as a unit. The writing is generally clear and precise, and the numerous illustrations provide an easier understanding of the phenomena described. The volume contains 20 Chapters covering altogether many (although not all) semiconductors of technological interest, starting with the IV-IV group compounds ( $\mathrm{SiC}$ and SiGe), carrying on with the binary and ternary compounds of the III-V (GaAs, AIGaAs, GaSb, InAs, GaP, InP, and GaN) and II-VI ( $\mathrm{HgTe}, \mathrm{HgCdTe}$ ) families, the metal oxides ( $\mathrm{CuO}, \mathrm{ZnO}, \mathrm{ZnCoO}$, tungsten oxide, and $\mathrm{PbTiO}$ ), and finishing with $\mathrm{Bi}$ (a semimetal).

\title{
How to reference
}

In order to correctly reference this scholarly work, feel free to copy and paste the following:

Takahiro Shimada and Takayuki Kitamura (2010). Multi-Physics Properties in Ferroelectric Nanowires and Related Structures from First-Principles, Nanowires, Paola Prete (Ed.), ISBN: 978-953-7619-79-4, InTech, Available from: http://www.intechopen.com/books/nanowires/multi-physics-properties-in-ferroelectricnanowires-and-related-structures-from-first-principles

\section{INTECH}

open science | open minds

InTech Europe

University Campus STeP Ri

Slavka Krautzeka 83/A

51000 Rijeka, Croatia

Phone: +385 (51) 770447

Fax: +385 (51) 686166

www.intechopen.com
InTech China

Unit 405, Office Block, Hotel Equatorial Shanghai

No.65, Yan An Road (West), Shanghai, 200040, China

中国上海市延安西路 65 号上海国际贵都大饭店办公楼 405 单元

Phone: +86-21-62489820

Fax: +86-21-62489821 
(C) 2010 The Author(s). Licensee IntechOpen. This chapter is distributed under the terms of the Creative Commons Attribution-NonCommercialShareAlike-3.0 License, which permits use, distribution and reproduction for non-commercial purposes, provided the original is properly cited and derivative works building on this content are distributed under the same license. 